\title{
Georges Lemaître and Stigler's Law of Eponymy
}

\author{
David L. Block
}

\begin{abstract}
One of the greatest discoveries of modern times is that of the expanding Universe, almost invariably attributed to Hubble (Proceedings of the National Academy of Sciences of the United States of America 15:168, 1929). What is not widely known is that the original treatise by Lemaitre (Annales de la Société Scientifique de Bruxelles, Sèrie A 47:49, 1927) contained a rich fusion of both theory and of observation. The French paper was meticulously censored when published in English: all discussions of radial velocities and distances, and the very first empirical determination of $H_{0}$, were suppressed. Stigler's law of eponymy is yet again affirmed: no scientific discovery is named after its original discoverer (Merton, American Sociological Review 22(6):635, 1957). An appeal is made for a Lemaitre Telescope naming opportunity, to honour the discoverer of the expanding universe.
\end{abstract}

\section{Lemaître (1927): A Theoretical Paper?}

The title of the original 1927 paper indicates to the reader that the content will be a fusion of both theory and of observation: "Un univers homogène de masse constante et de rayon croissant, rendant compte de la vitesse radiale des nébuleuses extra-galactiques." Which translates into English thus: "A homogeneous universe of constant mass and increasing radius accounting for the radial velocity of extragalactic nebulae".

Lemaitre spent the years 1924-1925 at the Harvard College Observatory. He had an excellent foundation in observational astronomy, writing about terms such as the effective temperatures of stars, trigonometric parallaxes, moving-cluster parallaxes, absolute bolometric magnitudes, dwarf branch stars, giant branch stars, and the like.

\footnotetext{
D.L. Block $(\bowtie)$

School of Computational and Applied Mathematics, University of the Witwatersrand, Johannesburg, South Africa

e-mail: david.block@wits.ac.za
}

R.D. Holder and S. Mitton (eds.), Georges Lemaittre: Life, Science and Legacy, Astrophysics and Space Science Library 395, 
To speak of Lemaître (1927) as a most remarkable and absolutely brilliant theoretical paper only, is a grave injustice to the very title. Not only does Lemaitre derive a linear relationship between the radial velocities of galaxies and their distances in the above paper, but he is eager to determine the rate at which the universe expands. Lemaître (1927) carefully uses the radial velocities of 42 extragalactic nebulae tabulated by Strömberg (1925), and he converts apparent magnitudes $m$ into distance $[\log r=0.2 m+4.04]$ following Hubble (1926). The actual value which Lemaitre obtains in 1927 for the rate of expansion of the Universe is $625 \mathrm{~km} \mathrm{~s}^{-1} \mathrm{Mpc}^{-1} ; 575 \mathrm{~km} \mathrm{~s}^{-1} \mathrm{Mpc}^{-1}$ with different weighting factors (Fig. 1).

Jaki (1974) elaborates: “Lemaître's treatment of the problem could hardly be more impressive with respect to specific results ... a formula and a table of values for the redshift of receding galaxies in fine agreement with the actually observed data ..."

When the Royal Astronomical Society decided to publish an English translation in 1931 from the journal Annales de la Société Scientifique de Bruxelles, a most dramatic censorship of the first empirical determination of $H_{0}$ occurred (Fig. 2). A meticulously researched book (with a foreword by the late Allan Sandage) has been published on this precise theme. It is entitled Discovering the Expanding Universe (Nussbaumer and Bieri 2009). Professor Nussbaumer graciously sent me a copy of the original French paper in 2009, and the sectors censored out in the English translation appear in Fig. 2. Equation (24) holds the key. In an independent study, Sidney van den Bergh (2011) affirms that the suppressions in Eq. (24) were intentional.

It would be historically accurate to say that the testing of a linear velocitydistance relation is due to the meticulous observations by Hubble and Humason in subsequent years, but not the formulation of this relation, as seen in the complete original equation (24).

\section{Priorities in Scientific Discovery}

And now, I give some insight into the mindset of Edwin Hubble. He was fiercely territorial, as we see in a letter from Hubble to de Sitter, dated 21 August 1930, wherein Hubble writes: "I consider the velocity-distance relation, its formulation, testing and confirmation, as a Mount Wilson contribution and I am deeply concerned in its recognition as such" (emphasis added).

Nussbaumer and Bieri (2009) respond as follows:

... the formulation and its central place in cosmology was first given by Lemaitre ... there is no justification to glorify Hubble's publication of 1929 [as the] original discovery of the linear velocity- distance relationship ... (emphasis, mine).

Lemaitre was eclipsed. Multitudes of textbooks proclaim Hubble as the discoverer of the expanding universe. But herein lies a repeated pattern. In 1927, 

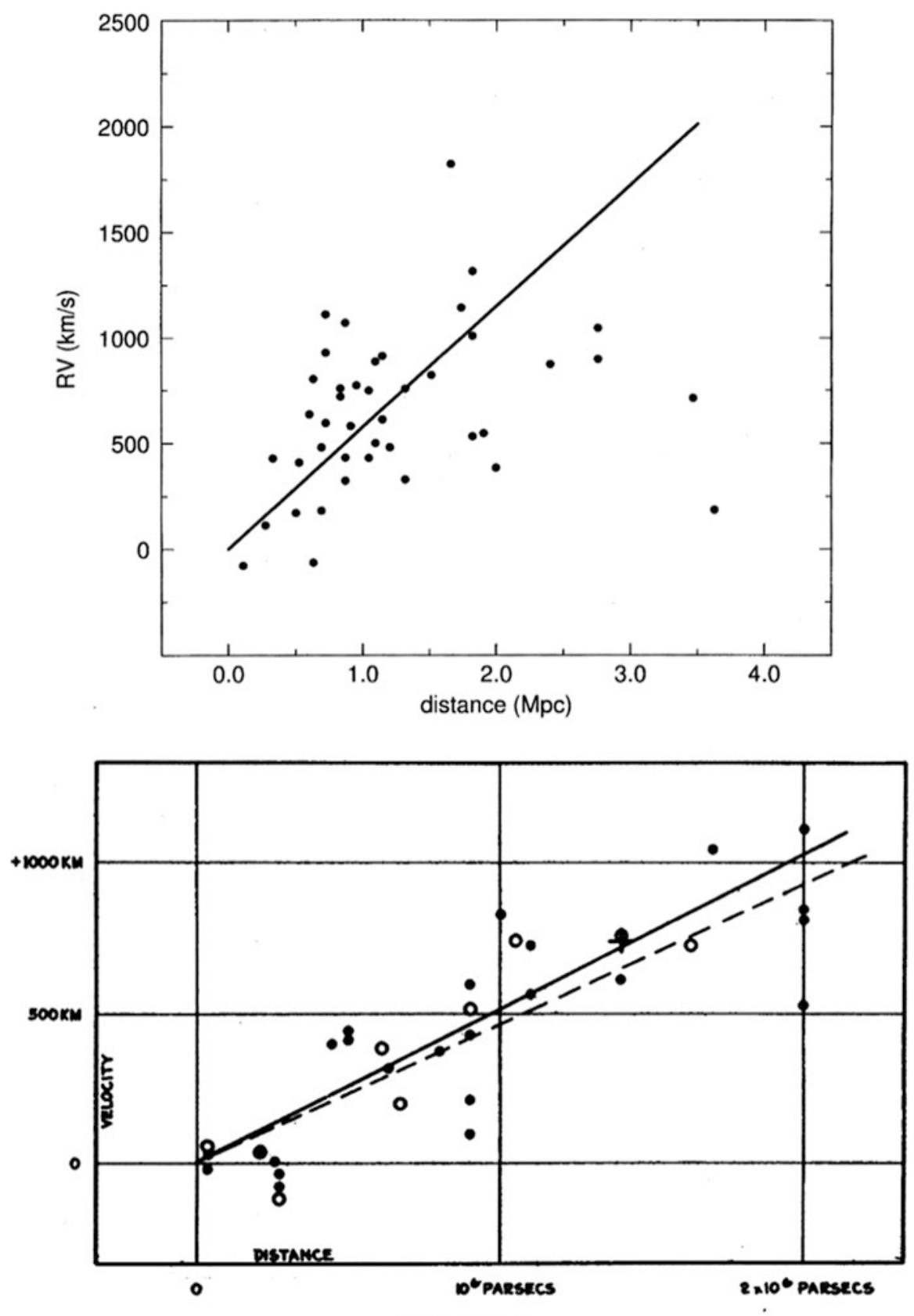

FIGURE 1

Fig. 1 Upper panel: The data used by Lemaitre (1927) to yield the first empirical value of the rate of expansion of the Universe in which $v / r$ is predicted to be constant (see Eq. 24 in Fig. 2). Lemaitre derived values of $625 \mathrm{~km} \mathrm{~s}^{-1} \mathrm{Mpc}^{-1}$ and $575 \mathrm{~km} \mathrm{~s}^{-1} \mathrm{Mpc}^{-1}$. The solid line in the top panel has a slope of $575 \mathrm{~km} \mathrm{~s}^{-1} \mathrm{Mpc}^{-1}$ and is reconstructed by H. Duerbeck. Lower panel: the radial velocity-distance diagram published by Hubble, 2 years later, in 1929, with best slope of $530 \mathrm{~km} \mathrm{~s}^{-1} \mathrm{Mpc}^{-1}$ (Top panel: Courtesy H. Duerbeck) 


$$
-s s-
$$

période de la lumière reçue et $\mathrm{Ot}_{4}$ peut encore étre considéré comme la période d'une lumiêre émise dans les mémes conditions dans le voisinage de l'observateur. En effet, la période de la lumière émise dans des conditions physigues semblables doit etre partout la méme lorsqu'elle est exprimée en temps propre.

$$
\frac{v}{c}-\frac{\partial t_{1}}{\partial t_{1}}-1-\frac{\mathrm{R}_{2}}{\mathrm{H}_{4}}-1
$$

mesure donc l'effet Doppler apparent dû a la variation du rayon de l'univers. Il est egal a lexdes sür liunile du rapport des rayons de lunivers d l'instant ou la lumizre est reçue et à linstant où elle est tmise. $v$ est la vitesse de l'observateur qui produirait le mème effet. Lorsque la source est suffisamment proche nous pouvons écrire approximativement

$$
\frac{v}{c}-\frac{\mathrm{R}_{1}-\mathrm{R}_{1}}{\mathrm{R}_{\mathrm{a}}}-\frac{d \mathrm{R}}{\mathrm{R}}-\frac{\mathrm{R}^{\prime}}{\mathrm{R}} d t-\frac{\mathrm{R}^{\prime}}{\mathrm{R}},
$$

oủ $r$ est la distance de la source. Nous avons donc

$$
\frac{\mathrm{R}^{\prime}}{\mathrm{R}}=\frac{v}{\mathrm{cr}}
$$

Les vitesses radiales de $4 \mathbf{3}$ nébuleuses extra-galactiques sont données parmplerg ('). quabely rese galactiques sont de grandeurs absolues sensibteteindre $-15,2310$ parsecs, les ecarts individuels pouvant en parsecs esvig dd ne palla formule log $r=0,2 m+4,04$.

On trouve une dist 17 dd pero de $10^{4}$ parsecs, variant de quelques dixièmes à 3,3 millid ar probable résultant de la dispersion en grandeur $x$ tolug es diférence de grandeur absolu fux gras en plus ou en moins,

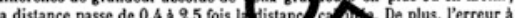

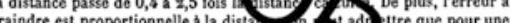
caindre est proportionnellea la distam oun adtre que pour une crance dun persion en (a) propor ane aux aébuleuses. On peut espérer éviter une erreur donnant aux observations un poids proportionnel a $\frac{1}{\sqrt{1+r^{2}}}$ distance en millions de parsecs.

(i) Analynis of radial relocities of globular clasters and non galactic nebulae. Ap. J.

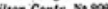

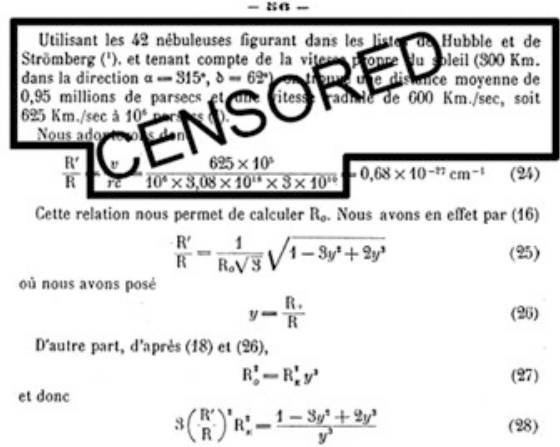

. Introduisant les valeurs numériques de $\frac{R^{\prime}}{R}(26)$ et de $R_{\varepsilon}(19)$, il vient :

$y=0,0465$.

$\mathrm{R}-\mathrm{R}_{\mathrm{s}} \mathrm{V} \overline{\mathrm{y}}-0,215 \mathrm{R}_{\mathrm{x}}-1,83 \times 10^{\prime \prime} \mathrm{cm},-6 \times 10^{\prime}$ parsecs

$\mathrm{R}_{0}=\mathrm{R}_{\mathrm{y}}=\mathrm{R}_{\mathrm{s}} \mathrm{yi}^{3}-8,5 \times 10^{\prime \prime} \mathrm{cm} \cdot-2,7 \times 10^{\circ}$ parsecs

$-9 \times 10^{*}$ années de lumiére.

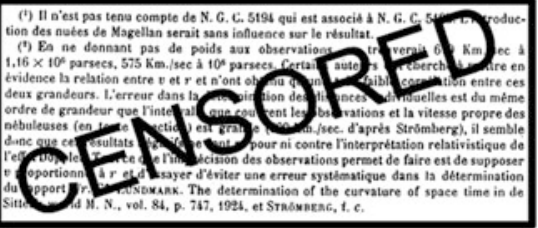

Fig. 2 Sections in black boxes, pertaining to the discussion and use of radial velocities of galaxies and their distances by Lemaitre (1927) to provide the first empirical determination of $H_{O}$ were meticulously and ingeniously suppressed or censored in the English translation. Equation (24) is absolutely crucial

Knut Lundmark penned these words, cited by Sandage (2004): “As to Hubble's way of acknowledging his predecessors I have no reason to enter upon this question here."

Is it not strange that Vesto Slipher is not referenced at all in Hubble's landmark paper of 1929? The vast majority of radial velocities in that paper are from Slipher. Perhaps an even more glaring example is Fig. 3, written to J. H. Reynolds on a visit to England.

As elucidated by Block and Freeman (2008), Reynolds rises to the Hubble request. He publishes his results in Reynolds (1920). Hubble very carefully studied this paper and actually pencilled in some handwritten comments, shown to me by the late Allan Sandage. (For example, next to each of the Reynolds class II, III and IV are the $\mathrm{Sa}, \mathrm{Sb}$ and $\mathrm{Sc}$ notations penciled in by Hubble. Dr. Sandage furthermore affirmed to me that the correspondence between Reynolds types and Hubble types is "one-to-one"). Hubble (1926) appeared in print 6 years after Reynolds - with no reference to Reynolds (1920). Was Lundmark correct?

In the English speaking world, a total eclipse fell on the remarkable astronomical insight of Lemaître (Kragh and Smith 2003). The translator has been demonstrated to be Lemaître himself (see below my Note Added in Proof). What an intriguing 
Georges Lemaitre and Stigler's Law of Eponymy

Could you nat thou you ideas into the form 2 a precise dassifinat 20 we coned actually apply it to a large number of nebulae representing the various sizes and degrees f brightness with which we will be dealing?

Y the are only passing thought which Is fer. The great thin is that the discussion is slanted. I his will comblually lead to sonicthing acepplatile to us all.

Sincerely

Edwin Hubble.

Aches after Saturday

initio toted

axing.

Fig. 3 Hubble requests the following from J. H. Reynolds: "Could you not throw your ideas into the form of a precise classification so we could actually apply it to a large number of nebulae representing the various sizes and degrees of brightness with which we will be dealing?" The letter is believed to have been written in 1919, a year in which Hubble is recorded to have dined in England. This letter was first reproduced in Block and Freeman (2008). The original is in the archives of the Royal Astronomical Society of London

proof of Stigler's Law of Eponymy; Lemaitre was, through his own actions, robbed of being attributed with one of the greatest discoveries in astronomy of all time. There are myriads of speculations as to why Lemaitre decided to omit his empirical computation of the rate of expansion of the universe from the English 


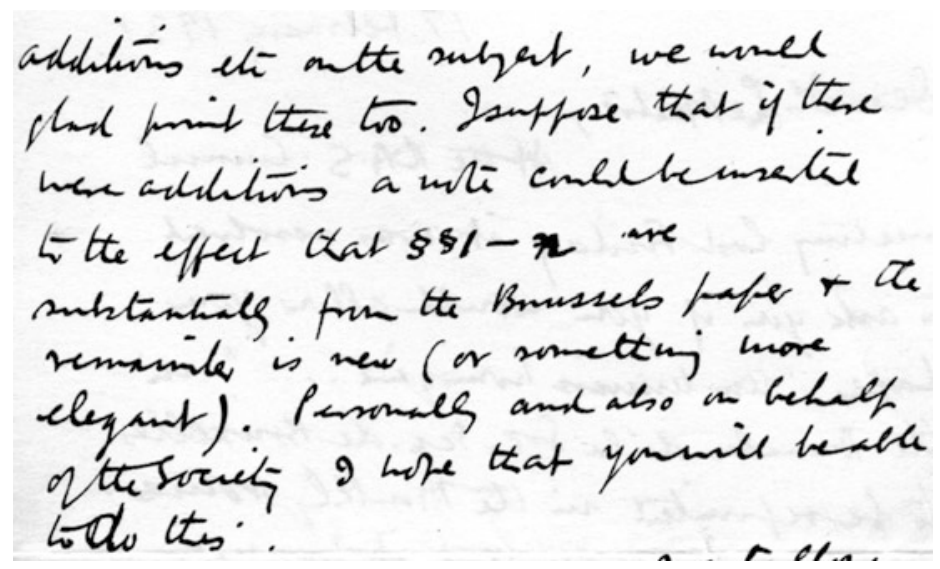

Fig. 4 The alarming "presence of a censor" is seen in this February 1931 letter from WM Smart to G. Lemaître. In extremely polite terms, Lemaître is told by Smart that Hubble's observational result of 1929 is "something more elegant". The reason we know that Smart is specifically alluding to Hubble (1929) is as follows: Lemaître is given full freedom to translate his 1927 French paper, from paragraph 1 to paragraph 72 (which at first glance, appears as a symbol "n", but which is actually the number " 72 " as affirmed by D. Lambert - private communication). Here follows the punch-line: paragraph 73 is Lemaître's equation 24. Paragraph 73 would have been the empirical determination by Lemaître of his expansion coefficient, published in 1927 (Courtesy: Lemaître Archives, Louvain-la-Neuve)

translation of his monumental 1927 paper (Fig. 4), although historians of astronomy must never forget his original intentions, as recalled by Lemaître himself several years later (in 1950, see below). The history is not irrelevant.

\section{CODA: A Lemaître ELT?}

One of Galileo's masterful works was entitled Sidereus Nuncius - the starry messenger. The moral of the censorship (Fig. 2) is - as Martin Gaskell (private communication) poignantly reminded me - Mark chapter 4, verse 22. I allow Nussbaumer and Bieri (2009) to have the final word here regarding the legendary Georges Lemaître: "Even in his influential The Realm of the Nebulae published in 1936, he [Hubble] avoided any reference to Lemaître. Was he afraid that a gem might fall from his crown if people became aware of Lemaitre's pioneering fusion of observation and theory 2 years before Hubble delivered the confirmation?" (italics, mine).

Acknowledgements First and foremost, I thank my co-author of Shrouds of the Night, K. C. Freeman, for his invaluable insight, encouragement and support. I am indebted to Harry Nussbaumer, Robert Smith and Sidney van den Bergh for their detailed comments on the manuscript. I warmly thank Dominique Lambert, Piet vd Kruit, Maarten Schmidt and the Director General of ESO, Tim de Zeeuw for their insight and interest. Profound appreciation goes to my sponsors AVENG and AECI for financial support and to archivist Mrs. Liliane Moens at the Lemaître Archives. 


\title{
Note Added in Proof - "The History of This Science Competition Is Not Irrelevant" - Reflections by Lemaître Himself, in 1950
}

The world has before its eyes one of the most brilliant examples of Stigler's law of eponymy -which in its simplest form, asserts that: no scientific discovery is named after its original discoverer. "Priorities in Scientific Discovery: A Chapter in the Sociology of Science" (Merton 1957) is of crucial importance in this context.

In a Comment published in Nature Mario Livio (Nature, 479, 171, 2011) has unearthed a letter from Lemaître to W. M. Smart (dated 9 March 1931). From that document, it is clear that Lemaitre himself translated his 1927 paper into English and who also omitted his determination of the coefficient of expansion of the Universe $\left(H_{0}\right)$ from values of radial velocities available as of 1927 . However, in his Comment Livio omits a vital reference, namely thoughts penned by Lemaître himself in 1950 (L'expansion de l'Univers, Bibliographie: Annales d'Astrophysique, 13, 344):

\begin{abstract}
About my contribution of 1927, I do not want to discuss if I was a professional astronomer. I was, in any event, an IAU member (Cambridge, 1925), and I had studied astronomy for two years, a year with Eddington and another year in the U.S. observatories. I visited Slipher and Hubble and heard him in Washington, in 1925, making his memorable communication about the distance [to] the Andromeda nebula. While my Mathematics bibliography was seriously in default since I did not know the work of Friedmann, it is perfectly up to date from the astronomical point of view; I calculate [in my contribution] the coefficient of expansion (575 km per sec per megaparsecs, 625 with a questionable statistical correction). Of course, before the discovery and study of clusters of nebulae, there was no point to establish the Hubble law, but only to calculate its coefficient. The title of my note leaves no doubt on my intentions: A Universe with a constant mass and increasing radius as an explanation of the radial velocity of extra-galactic nebulae. I apologize that all of this is too personal. But, as noted by the author (p. 161) "the history of this science competition is not irrevelant" and it is useful to highlight the details to enable an exact understanding of the scope of the argument that can be drawn from this. (Emphasis added)

In 1950, Lemaitre clearly did not want the rich fusion of theory and observations contained in his 1927 paper to be buried in the sands of time.
\end{abstract}

\section{References}

Block, D. L., \& Freeman, K. C. (2008). Shrouds of the night. New York: Springer.

Hubble, E. (1926). Extra-galactic nebulae. The Astrophysical Journal, 64, 321.

Hubble, E. (1929). A relation between distance and radial velocity among extra-galactic nebulae. Proceedings of the National Academy of Sciences of the United States of America, 15, 168.

Jaki, S. L. (1974). Science and creation. Edinburgh/London: Scottish Academic Press.

Kragh, H., \& Smith, R. W. (2003). Who discovered the expanding universe? History of Science, $41,141$.

Lemaître, G. (1927). Un univers homogène de masse constante et de rayon croissant, rendant compte de la vitesse radiale des nébuleuses extra-galactiques. Annales de la Société Scientifique de Bruxelles, Serrie A, 47, 49 [Translated into English in Monthly Notices of the Royal Astronomical Society, 91, 483].

Merton, R. K. (1957). Priorities in scientific discovery: A chapter in the sociology of science. American Sociological Review, 22(6), 635. 
Nussbaumer, H., \& Bieri, L. (2009). Discovering the expanding universe. Cambridge: Cambridge University Press.

Reynolds, J. H. (1920). Photometric measures of the nuclei of some typical spiral nebulae. Monthly Notices of the Royal Astronomical Society, 80, 746.

Sandage, A. (2004). Centennial history of the Carnegie Institution of Washington: Volume 1. The Mount Wilson Observatory. Cambridge: Cambridge University Press.

Strömberg, G. (1925). Analysis of radial velocities of globular clusters and non-galactic nebulae. The Astrophysical Journal, 61, 353.

Van den Bergh, S. (2011). The curious case of Lemaitre's equation no. 24. Journal of the Royal Astronomical Society of Canada, 105, 151. 